\title{
ГИПЕРКАЛЬЦИЕМИЧЕСКИЙ КРИЗ
}

\section{Калинин А.П. ${ }^{1}$, Котова И.В. ${ }^{1}$, Бритвин Т.А. ${ }^{1}$, Алаев Д.С. ${ }^{2}$, Белошицкий М.Е. ${ }^{1}$}

${ }^{1}$ ГБУЗ МО «Московский областной научно-исследовательский клинический институт им. М.Ф. Владимирского» (МОНИКИ); 129110, г. Москва, ул. Щепкина, 61/2, Российская Федерация

2ГБУЗ города Москвы «Городская клиническая больница №12 Департамента здравоохранения города Москвы» (ГБУЗ «ГКБ №12 ДЗМ»); 115516, г. Москва, ул. Бакинская, 26, Российская Федерация

В настоящей работе представлены особенности анамнеза, клиники, предоперационной подготовки, хирургического вмешательства, послеоперационного периода у больных с гиперкальциемическим кризом, развивающимся на фоне первичного гиперпаратиреоза (ПГПТ). В 2000-2013 гг. из 214 пациентов с ПГПТ в связи с развивающимся гиперкальциемическим кризом оперированы пять больных. Из ниху трех при гистологическом исследовании верифицирована паратиреоаденома, у одного - гиперплазия околощитовидных желез (ОЩЖ), у одного - рак ОЩЖ. У четырех из пяти пациентов ПгПТ впервые диагностирован на фоне гиперкальциемического криза. У одной пациентки ПГПТ выявлен «на фоне» беременности 25-26 недель. В связи с абдоминальной симптоматикой (боли в животе, рвота), характерной для гиперкальциемического криза, у нее проводилась ревизия брюшной полости в Центральной районной больнице, а диагноз ПГПТ был поставлен позже в отделении абдоминальной хирургии МОНИкИ. Еще у одной пациентки, которая наблюдалась по месту жительства по поводу артрита, уровень паратиреоидного гормона (ПТГ) перед операцией достигал 6490 пг/мл, кальция - 3,75 моль/л. В послеоперационном периоде у нее развилась не только острая почечная недостаточность, но и надпочечниковая. В одном наблюдении диагноз ПгПт был известен, уровни кальция и ПТГ не достигали высоких значений, гиперкальциемический криз развился на фоне операции на брюшной полости. Эта пациентка была оперирована на третьи сутки после первой операции, когда уже находилась в коме в связи с развившимся гиперкальциемическим кризом. Еще две пациентки поступили в клинику хирургической эндокринологии в тяжелом состоянии, у одной из них в течение нескольких лет ПГПТ принимали за ревматоидный артрит, миеломную болезнь, у другой отмечалась полиурия неясного генеза. У четырех из этих пяти больных в ближайшем послеоперационном периоде развились выраженные электролитные нарушения (снижение не только уровня кальция, но и калия, фосфора и магния), острая почечная недостаточность, полиорганная недостаточность, синдром диссеминированного внутрисосудистого свертывания. В ближайшем послеоперационном периоде умерли трое (на 22-, 32- и 34-е сутки). В связи с этим при выявленном ПГПТ операции по поводу данного заболевания и хирургические вмешательства на других органах необходимо проводить симультанно. Обязательным является контроль уровней креатинина, кальция, фосфора, магния, калия крови, контроль артериального давления каждые 2-3 часа, свертывающей системы крови в стационаре не менее 10 дней и еженедельно в течение 2-3 месяцев по месту жительства.

Ключевые слова: первичный гиперпаратиреоз, околощитовидные железы, гиперкальциемический криз.

\section{HYPERCALCEMIC CRISIS}

\section{Kalinin A.P. ${ }^{1}$, Kotova I.V. ${ }^{1}$, Britvin T.A. ${ }^{1}$, Alaev D.S. ${ }^{2}$, Beloshitskiy M.E. ${ }^{1}$}

${ }^{1}$ Moscow Regional Research and Clinical Institute (MONIKI); 61/2 Shchepkina ul., Moscow, 129110, Russian Federation ${ }^{2}$ Municipal Clinical Hospital No.12 (MCH No.12), Moscow Department of Public Health; 26 Bakinskaya ul., Moscow, 115516, Russian Federation

The paper presents the peculiarities of history, clinic, presurgical preparation, surgical intervention, and postoperative period in patients with hypercalcemic crisis caused by primary hyperparathyroidism (PHPT). During 2000-2013, 5 of 214 patients with PHPT who developed hypercalcemic crisis were operated on. In 3 of them, histological analysis verified parathyroid adenoma, in 1 - parathyroid hyperplasia, and in 1 - parathyroid carcinoma. In 4 of 5 patients, PHPT was first revealed against the background of hypercalcemic crisis. In one female patient, PHPT was found "against the background" of 25-26-week pregnancy. Due to abdominal symptoms (abdominal pain, vomiting) characteristic of the hypercalcemic crisis, she underwent abdominal cavity revision in the Central Municipal Hospital. PHPT was diagnosed later, in the MONIKI Department of Abdominal Surgery. In the other female patient who was observed at the place of her residence for arthritis, the level of parathyroid hormone (PTH) before surgery reached $6490 \mathrm{pg} / \mathrm{ml}$, and calcium $-3.75 \mathrm{~mol} / \mathrm{L}$. In the postoperative period, she developed not only acute renal but also adrenal failure. In one patient with known PHPT and not very high calcium and PTH levels, hypercalcemic crisis developed against the background of abdominal surgery. This female patient was operated on in two days after the first operation because she was in coma due to developed hypercalcemic crisis. Two more female patients were admitted to the clinic of endocrine surgery in severe condition. In one of them, PHPT was mistaken for rheumatoid arthritis or myeloid disease for several years, in the other, polyuria of unclear genesis was noted. In 4 of these 5 patients, in the nearest postoperative period, marked electrolytic disturbances developed (decreased levels of calcium, potassium, phosphorus, and magnesium) as well as an acute renal failure, polyorganic insufficiency, and syndrome of disseminated intravascular blood coagulation. In the nearest postoperative period, three patients died (on the $22^{\text {nd }}, 32^{\text {nd }}$, and $34^{\text {th }}$ day). In this connection, in patients with revealed PHPT, the surgical operations for the given disease as well as on the other organs should be performed simultaneously. The levels of creatinine, calcium, phosphorus, magnesium, and blood potassium should be under the control as well as an arterial pressure (every 2-3 hours), and blood coagulation (not less than during 10 days and then every week during 2-3 months at the place of residence).

Key words: primary hyperparathyroidism, parathyroid glands, hypercalcemic crisis. 
Гиперкальциемический криз - тяжелое осложнение первичного гиперпаратиреоза (ПГПТ), возникающее при резком повышении уровня кальция в крови. При этом полной корреляции между уровнем кальция и тяжестью криза может не быть [1, 2, $3,4,5]$. Повышение уровней общего кальция до 3,5 ммоль/л, ионизированного кальция до 1,8 ммоль/л свидетельствует о начале развития криза. Гиперкальциемический криз - одна из самых частых причин летальных исходов при ПгПт (5760\% наблюдений) [6]. Как правило, гиперкальциемический криз развивается у пациентов, длительно страдающих ПГПТ. У них заболевание сопровождается дегидратацией, а также последствиями воздействия паратиреоидного гормона (ПТГ) на органы-мишени (нефролитиаз, нефрокальциноз, костная резорбция) [7]. Вследствие снижения скорости клубочковой фильтрации и задержки кальция развивается тяжелая гиперкальциемия [8]. Основными симптомами гиперкальциемического криза являются спутанность сознания (вплоть до комы), боли в животе, тошнота, рвота, полиурия, а при прогрессировании криза - анурия.

Цель исследования - анализ собственных наблюдений гиперкальциемического криза у больных с ПГПТ.

В 2000-2013 гг. в отделении хирургической эндокринологии МОНИКИ по поводу ПГПТ оперированы 214 пациентов в возрасте 14-72 лет, из них пять - по неотложным показаниям в связи с гиперкальциемическим кризом. У трех при гистологическом исследовании верифицирована паратиреоаденома,уодного-гиперплазияоколощитовидных желез (ОЩЖ), у одного - рак ОЩЖ. Определялись уровни общего и ионизированного кальция, ПТГ, проводились ультразвуковое исследование (УЗИ), компьютерная томография (КT). Сцинтиграфия ОЩж с Технетрилом не проводилась в связи с тяжестью состояния пациентов (невозможностью их доставки в лечебное учреждение, в котором проводится исследование).

Приводим клинические наблюдения.
1. У пациентки Б., 23 года, ПгПТ диагностирован «на фоне» беременности 25-26 недель. Больная поступила в стационар по месту жительства на 15-й неделе беременности с жалобами на острые боли в эпигастральной области и рвоту. С подозрениями на панкреонекроз на вторые сутки оперирована: выполнены верхне-срединная лапаротомия, ревизия брюшной полости, дренирование сальниковой сумки, забрюшинного пространства и брыжейки. Послеоперационное течение - тяжелое. По данным УзИ - острый панкреатит, реактивный левосторонний плеврит, кальцификация плаценты. Переведена в отделение абдоминальной хирургии МОНИКИ. В связи с гиперкальциемией (3,6 ммоль/л), гипофосфатемией (0,84 ммоль/л) консультирована эндокринологом. При Узи обнаружено новообразование в проекции левой нижней ОЩж. Уровень ПтГ - 339 пг/мл. Для подготовки к операции в течение двух суток проводилась коррекция электролитных нарушений - восполнение дефицита калия, магния; по поводу угрозы прерывания беременности получала Гинипрал; в связи с выявлением эрозий желудка и двенадцатиперстной кишки и с целью предотвращения развития желудочно-кишечного кровотечения вводился омепразол. Операция: удаление аденомы левой нижней ОЩЖ. В послеоперационном периоде уровень общего и ионизированного кальция оставался повышенным в течение трех суток, на четвертые сутки развилась гипокальциемия, прогрессировали гипокалиемия, гипомагниемия, уровень креатинина был в пределах нормальных значений. Послеоперационное состояние корректировалось назначением препаратов кальция, магния, омепразола. Гистологически: аденома ОЩж. Роды срочные (кесарево сечение), ребенок здоров.

2. Пациентку Б., 55 лет, в течение двух лет лечили по месту жительства от предположительного артрита. При поступлении в МОНИКИ больная самостоятельно не передвигалась. Уровень ПтГ - 6490 пг/мл, кальция 3,75 моль/л, креатинина - 152 мкмоль/л, калия 4,2 ммоль/л, фосфора - 1,0 ммоль/л, магния 0,66 ммоль/л, гемоглобина - 82,0 г/л. При УзИ обнаружено узловое образование (паратиреоаденома?) за нижним полюсом левой доли щитовидной железы. В течение трех суток проводилась инфузионная терапия, затем по экс-

Калинин Ариан Павлович - чл.-кор. РАН, д-р мед. наук, профессор, вед. науч. сотр. отделения хирургической эндокринологии МОНИКИ. Котова Ирина Владимировна - д-р мед. наук, вед. науч. сотр. отделения хирургической эндокринологии МОНИКИ. Бритвин Тимур Альбертович - д-р мед. наук, руководитель отделения хирургической эндокринологии МОНИКИ. Алаев Дмитрий Сергеевич врач-хирург хирургического отделения №1, ГБУз «ГКБ №12 ДЗМ». Белошицкий Михаил Евгеньевич - д-р мед. наук, ст. науч. сотр. отделения хирургической эндокринологии МОНИКИ.

Для корреспонденции: Бритвин Тимур Альбертович - 129110, Москва, ул. Щепкина, 61/2, Российская Федерация. Тел.: +7 (495) 6317283. E-mail: t.britvin@gmail.com

Kalinin Arian Pavlovich - Corr. member of RAS, MD, PhD, Professor, leading scientific worker, Department of Surgical Endocrinology, MONIKI. Kotova Irina Vladimirovna - MD, PhD, leading researcher of the Department of Surgical Endocrinology, MONIKI. Britvin Timur Al'bertovich MD, PhD, Head of the Department of Surgical Endocrinology, MONIKI. Alaev Dmitriy Sergeevich - the surgeon of the Surgical Department No.1, MCH No.12. Beloshitskiy Mikhail Evgen'evich - MD, PhD, senior researcher, Department of Surgical Endocrinology, MONIKI.

Correspondence to: Britvin Timur Al'bertovich - 61/2 Shchepkina ul., Moscow, 129110, Russian Federation. Tel.: +7 (495) 63172 83. E-mail: t.britvin@gmail.com 
тренным показаниям проведена операция: удалена паратиреоаденома левой нижней ОЩж. Гистологически: ОЩж в тонкой фиброзной капсуле, с диффузной гиперплазией преимущественно главных светлых и темных клеток, с образованием трабекулярных и микрофолликулярных структур. На вторые сутки после операции - резкое снижение артериального давления до 60/40 мм рт. ст., которое не купировалось введением глюкокортикоидов, гемоглобин с 80,0 снизился до 67 г/л в течение двух часов. В отделении реанимации проводились гемотрансфузия, терапия глюкокортикоидами, минералокортикоидами (Кортинеф), Дофамином, препаратами кальция, Фосфалюгелем. Биохимические показатели: ионизированный кальций -1,16-1,00-0,93-0,86; фосфор-0,37-0,38-0,48; калий - 3,2 - 5,1; креатинин - 299 - 317 - $259-208$ - 101, магний - 0,7; ПтГ - 32-44 пг/мл. Развилась полиорганная недостаточность (в том числе надпочечниковая), характеризовавшаяся стойкой гиподинамией, «острым» почечным синдромом, развившимся на фоне хронической болезни почек, электролитными нарушениями, прогрессирующей анемией. В настоящее время состояние пациентки удовлетворительное, стабильное, электролитные нарушения не отмечаются.

3. Пациентка С., 52 года, которую в стационаре по месту жительства обследовали по поводу предполагаемого ревматоидного артрита, миеломной болезни, поступила в отделение хирургической эндокринологии МОНИКИ в тяжелом состоянии. В правой доле щитовидной железы пальпировалось узловое образование до 2 см в диаметре без четких контуров. Лабораторные данные: гемоглобин - 73 г/л, белок - 62 г/л, креатинин - 131 мкмоль/л, общий кальций - 3,54 ммоль/л, ионизированный кальций2,12, щелочная фосфатаза - 306 ед/л, птГ - 1640 пг/мл. КТ шеи: изменение структуры правой доли щитовидной железы с визуализацией многоузлового образования; небольшая мягкотканная структура вдоль правых отделов пищевода; с учетом клиники не исключается увеличение эктопированной ОЩж. Системное изменение структуры костей обусловлено ПгПт. Учитывая высокий риск возникновения гиперкальциемического криза, после проведения неотложной инфузионной терапии и форсированного диуреза в течение суток пациентка оперирована: удалена опухоль правой нижней Ощж. Гистологически: рак ОЩЖ. На вторые сутки развилась острая почечная недостаточность на фоне хронической болезни почек, по поводу которой проводился гемодиализ. Переведена в отделение терапевтической эндокринологии МОНИкИ для дальнейшего лечения. Уровень креатинина в послеоперационном периоде в динамике: 286 - 359 - 337 мкмоль/л; уровень калия - 3,84 ммоль/л. Уровень ПтГ снизился до 53 пг/мл, ионизированного кальция - до 0,93-0,94 ммоль/л. В заместительной почечной терапии пациентка не нуждалась. В удовлетворительном состоянии переведена в стационар по месту жительства. Через месяц повторно госпитализирована по месту жительства с клиникой почечной недостаточности, но от заместительной почечной терапии отказалась. Пациентка умерла на 64-й день после операции. Непосредственные причины смерти: хроническая почечная недостаточность, фибринозный перикардит.

4. Пациентка Л., 52 года, вначале поступила в отделение терапевтической эндокринологии по неотложным показаниям с жалобами на слабость, раздражительность, сухость во рту, жажду, полиурию (до 5 л/сут), никтурию, боли в костях ног, таза, поясничном отделе позвоночника, коленных суставах, снижение массы тела, ухудшение памяти, головные боли, приступы рвоты. Уровень Птг 600 пг/мл, кальция - 3,6 ммоль/л. Как было установлено, страдала ПгПт более 10 лет. После инфузионной терапии (до 6 л/сут), проводимой в течение трех суток, переведена в отделение хирургической эндокринологии. В связи с нарастанием клиники гиперкальциемического криза экстренно оперирована: удалены 3,5 ОЩЖ. Гистологически: гиперплазия всех ОЩЖ. На вторые сутки развилась гипокальциемия (ионизированный кальций - 0,8 ммоль/л, калий - 3,6 ммоль/л, птГ - 16,5 пг/мл). Послеоперационный диагноз: состояние после удаления 3,5 ОЩЖ гиперкальциемического криза; синдром диссеминированного внутрисосудистого свертывания (ДВС-синдром) (1-я фаза); хронический панкреатит с экскреторной недостаточностью (стадия нестойкой ремиссии); железодефицитная анемия; энцефалопатия; полиневропатия. На 20-е сутки после операции в стационаре по месту жительства резко снизились уровни кальция и калия. На 22-е сутки на фоне полиорганной недостаточности наступила смерть от острой сердечно-сосудистой недостаточности.

5. У пациентки Е., 73 года, умершей на 32-й день после операции, при поступлении в отделение хирургической эндокринологии МОНИКИ уровень ПтГ составлял 248-364 пг/мл, ионизированного кальция - 1,78 ммоль/л, общего кальция - 2,99 ммоль/л. ПгПт не был диагностирован в течение 30 лет (в анамнезе шесть патологических переломов костей обоих предплечий, ишемическая болезнь сердца, артериальная гипертония, нефрокалькулез). Выявлено новообразование в брюшной полости неясного генеза 74 мм в диаметре. Так как отмечались лишь средние значения уровня ионизированного кальция и необходимость в срочной операции по поводу ПгпТ отсутствовала, пациентка переведена в отделение абдоминальной хирургии, где выполнена резекция желудка. По данным гистологического исследования - гастроинтестинальная стромальная опухоль. На третьи сутки после операции в отделении реанимации при проведении медикаментозной терапии возник пароксизм фибрилляции предсердий (частота сердечных сокращений - до 143193 уд/мин). Антиаритмическая терапия была неэффективной. Угнетение сознания до комы 1. Уровень кальция повысился до 3,35 ммоль/л, птг - до 370 пг/мл, гипертермия - до $39,0^{\circ} \mathrm{C}$. При УзИ паратиреоаденома в области шеи не визуализировалась. Несмотря на проведение 
инфузионной терапии и введение бисфосфонатов, гиперкальциемический криз нарастал. Экстренно проведены ревизия ОЩЖ, удаление эктопированной в параэзофагеальное пространство опухоли левой верхней ОЩЖ. Так как у пациентки был многоузловой нетоксический зоб 2-й степени (классификация ВОЗ), из-за которого возникли затруднения при удалении паратиреоаденомы, выполнена тиреоидэктомия. Гистологически: «старая» атипическая аденома ОЩж, микро-, макрофолликулярный коллоидный зоб, “старая» микро-макрофолликулярная аденома щитовидной железы. На пятые сутки при сохраняющихся гипертермии, гипоальбуминемии, нарушениях водно-электролитного баланса и гипокальциемии развились отек головного мозга, ДВС-синдром. Пациентка умерла на 32-й день после операции. Непосредственная причина смерти: сердечно-дыхательная недостаточность с развитием отека головного мозга.

Еще у одной пациентки гиперкальциемический криз развился в отделении урологии на фоне литотрипсии. Больная погибла в отделении реанимации. Диагноз пгПт был установлен при патоморфологическом исследовании.

На основании вышесказанного можно сделать вывод о том, что в предоперационном периоде всем пациентам с угрозой развития гиперкальциемического криза необходимо отменить все лекарственные средства, способствующие повышению уровня кальция крови, проводить регидратацию с целью нормализации экскреции кальция с мочой, форсированный диурез, при возможности - введение бисфосфонатов. Фактором, провоцирующим развитие гиперкальциемического криза, является хирургическое вмешательство по поводу заболеваний, сопутствующих ПГПТ. При выявленном ПГПТ операции по поводу данного заболевания и хирур- гические вмешательства на других органах необходимо проводить симультанно или после операции по поводу ПГПТ.

\section{Литература}

1. Калинин АП, Балаболкин МИ, Лукьянчиков ВС, Фексон ЭГ. Гиперкальциемический криз: методические рекомендации. М.: МОНИКИ; 1990. (Kalinin AP, Balabolkin MI, Luk'yanchikov VS, Fekson EG. Hypercalcemic crisis: methodical recommendations. Moscow: MONIKI; 1990. Russian).

2. Голохвастов НН, Рыбаков ГВ, Түробова ЕП, Яковлева ЕС. Гиперпаратиреоидный криз и некоторые факторы его развития. В: Современные аспекты эндокринологии: материалы 3-го (III) Российского симпозиума по хирургической эндокринологии. Самара; 1994. с. 130-6. (Golokhvastov NN, Rybakov GV, Turobova EP, Yakovleva ES. Hyperparathyroidism crisis and some factors of its development. In: Contemporary aspects of endocrinology: materials of the $3^{\text {rd }}$ (III) Russian symposium on Endocrine Surgery. Samara; 1994. p. 130-6. Russian).

3. Голохвастов НН, Рыбаков ГВ, Кацев ВМ, Дмитриева ЛА, Дундуков НН. Диагностическая и лечебная тактика при угрозе развития гиперпаратиреоидного криза. В: Хирургия эндокринных желез: материалы 5-го (VII) Российского симпозиума по хирургической эндокринологии. СПб.; 1996. с. 27-9. (Golokhvastov NN, Rybakov GV, Katsev VM, Dmitrieva LA, Dundukov NN. Diagnostic and therapeutic tactics in the threat of hyperparathyroidism crisis development. In: Surgery of endocrine glands: materials of the $5^{\text {th }}$ (VII) Russian symposium on Endocrine Surgery. St. Petersburg; 1996. p. 27-9. Russian).

4. Калинин АП, Нурманбетов ДН, Лукьянчиков ВС. Первичный гиперпаратиреоз. Бишкек: Илим; 1992. (Kalinin AP, Nurmanbetov DN, Luk'yanchikov VS. Primary hyperparathyroidism. Bishkek: Ilim; 1992. Russian).

5. Grossman RF, Jossart GH. Hypercalcemic crisis. In: Clark $\mathrm{OH}$, Duh QY, editors. Textbook of endocrine surgery. Phyladelphia: W.B. Saunders Company; 1997. p. 432-8.

6. Рожинская ЛЯ, Мокрышева НГ, Кузнецов СН. Алгоритмы обследования и лечения пациентов в эндокринологии. Часть II. Гиперпаратиреоз. М.; 2009. (Rozhinskaya LYa, Mokrysheva NG, Kuznetsov SN. Algorithms of examination and treatment of patients in Endocrinology. Part II. Hyperparathyroidism. Moscow; 2009. Russian).

7. Clark $\mathrm{OH}$, Duh QY. Primary hyperparathyroidism. A surgical perspective. Endocrinol Metab Clin North Am. 1989;18(3):701-14.

8. Секреты эндокринологии: учебное пособие. Пер. с англ. СПб.: Невский диалект; 2001. (Endocrine secrets: textbook. StPetersburg: Neva Dialect; 2001. Russian). 